\title{
Observation of GRBs and TGFs with AGILE
}

Francesco Longo $^{a, b}$, E. Del Monte ${ }^{c}$, M. Marisaldi $^{d}$, F. Fuschino ${ }^{d}$, E. Moretti ${ }^{e}$, A. Rappoldi ${ }^{f}$, A. Giuliani ${ }^{g}$, S. Cutini ${ }^{h}$, M. Trifoglio ${ }^{d}$, C. Pittori ${ }^{h}$, M. Tavani $^{c}, i$,

A. Argan ${ }^{c}$, G. Barbiellini ${ }^{a, b}$, E. Costa ${ }^{c}$, I. Donnarumma ${ }^{c}$, Y. Evangelista ${ }^{c}$, M. Feroci $^{c}$, M. Galli ${ }^{j}$, F. Gianotti ${ }^{d}$, C. Labanti ${ }^{d}$, I. Lapshov ${ }^{c, k}$, F. Lazzarotto ${ }^{c}$, S. Mereghetti ${ }^{g}$, L.Pacciani ${ }^{c}$, M.Rapisarda ${ }^{l}$, P. Soffitta ${ }^{c}$, A. Trois ${ }^{c}$, S. Vercellone ${ }^{m}$, F.Verrecchia ${ }^{h}$ on behalf of the AGILE team

${ }^{a}$ Dipartimento di Fisica, Università di Trieste, via A. Valerio 2, I-34127 Trieste, Italy

${ }^{b}$ INFN Trieste, via A. Valerio 2, I-34127 Trieste, Italy

${ }^{c}$ INAF-IASF Roma, via del Fosso del Cavaliere 100, I-00133 Roma, Italy

${ }^{d}$ INAF-IASF Bologna, via Gobetti 101, I-40129 Bologna, Italy

${ }^{e}$ Royal Institute of Technology (KTH), The Oskar Klein Centre for Cosmoparticle Physics, Stockholm, Sweden

${ }^{f}$ INFN Pavia, via Bassi 6, I-27100 Pavia, Italy

${ }^{g}$ INAF-IASF Milano, via E. Bassini 15, I-20133 Milano, Italy

${ }^{h}$ ASI Science Data Center, Via E. Fermi 45, I-00044 Frascati (Roma), Italy

${ }^{i}$ Dipartimento di Fisica, Università Tor Vergata, via della Ricerca Scientifica 1, I-00133 Roma, Italy

${ }^{j}$ ENEA, via Martiri di Monte Sole 4, I-40129 Bologna, Italy

${ }^{k}$ IKI, Moscow, Russia

${ }^{l}$ ENEA Frascati, via Enrico Fermi 45, I-00044 Frascati(Roma), Italy

${ }^{m}$ INAF-IASF Palermo, Via Ugo La Malfa 153, 90146 Palermo, Italy

E-mail: Erancesco.longo@ts.infn.it

The AGILE satellite, in orbit since 2007, localized up to October 2009 about 1 Gamma Ray Burst (GRB) per month with the hard X-ray imager SuperAGILE (18 - $60 \mathrm{keV}$ ) (with a rate reduced by a factor 2-3 in spinning mode) and is detecting around 1 GRB per week with the non-imaging Mini-Calorimeter (MCAL, $0.35-100 \mathrm{MeV}$ ). Up to now the AGILE Gamma Ray Imaging Detector firmly detected four GRBs in the energy band between $20 \mathrm{MeV}$ and few GeV. In this paper we review the status of the GRBs observation with AGILE and discuss the upper limits in the gamma-ray band of the non-detected events. Moreover AGILE is detecting hundreds of Terrestrial Gamma-ray Flashes (TGFs) with the MCAL instrument. Thanks to the MCAL energy range extended up to $100 \mathrm{MeV}$ and its flexible trigger logic on sub-millisecond time scales, AGILE is adding a wealth of observations which pose severe constrains on production models. In this presentation we will describe the characteristics of the AGILE TGF sample. Finally we present the first precise localization study of TGFs from space, carried out at gamma-ray energies above $20 \mathrm{MeV}$ with the AGILE gamma-ray imager.

25th Texas Symposium on Relativistic Astrophysics - TEXAS 2010

December 06-10, 2010

Heidelberg, Germany 


\section{The AGILE satellite}

Launched on April 2007, AGILE [1], [2] is a small scientific satellite of the Italian Space Agency (ASI) operating at a low inclination $\left(2.5^{\circ}\right)$ Low-Earth Orbit at $540 \mathrm{~km}$ altitude and is devoted to the observation of the sky in the hard X-ray and gamma-ray energy bands .

The AGILE payload is composed of two co-aligned imagers: the hard X-ray monitor SuperAGILE [3], sensitive in the 18-60 keV energy band with a field of view of $\sim 1 \mathrm{sr}$ and an angular resolution of 6 arcmin, and the Gamma Ray Imaging Detector (GRID, [4), a pair-tracking telescope based on a tungsten-silicon tracker [5], sensitive in the energy band from $30 \mathrm{MeV}$ to few $\mathrm{GeV}$ with a field of view of $\sim 2.5 \mathrm{sr}$ and a point spread function ranging between $3.5^{\circ}$ (at $100 \mathrm{MeV}$ ) and $1.5^{\circ}$ (at $1 \mathrm{GeV}$ ). The Minicalorimeter (MCAL, [6]) of the gamma-ray imager, based on CsI(Tl) scintillating bars, can independently detect transient events at $\mathrm{MeV}$ energies, using a dedicated trigger logic acting on several time scales spanning four orders of magnitude between $290 \mu \mathrm{s}$ and 8 seconds 河, in an almost all-Sky field of view with maximum sensitivity at an angle of roughly $90^{\circ}$ with respect to the satellite boresight.

\section{Observation of GRB with AGILE}

Both SuperAGILE and MCAL are equipped with on-board triggering algorithms (see [8] and [7] respectively) developed to detect short gamma-ray transient events such Gamma Ray Bursts (GRBs) and Terrestrial Gamma-ray Flashes (TGFs). Dedicated telemetry packets are introduced in the data stream to downlink the trigger information. Since the beginning of the AGILE operations, SuperAGILE and MCAL are active members of the InterPlanetary Network (IPN). On November 2009 AGILE suffered a malfunction to the reaction wheel and, after that time, the satellite is working in a spinning operative mode, with an angular velocity of $\sim 0.8^{\circ}$ per second around the axis pointed toward the Sun and scanning $\sim 70 \%$ of the Sky every orbit. In pointing operative mode the localization rate of SuperAGILE was $\sim 1$ GRB per month and it is now reduced of a factor of 2 - 3 in spinning mode. The MCAL detection rate is $\sim 1$ GRB per week and is not affected by the spinning operative mode. Until March 2011 the AGILE GRID firmly detected four GRBs in the gamma-ray band: GRB 080514B [9], [10], GRB 090401B [11], GRB 090510 [12], [13], [14] and GRB 100724B [15]. Two more GRBs (GRB 080721 and GRB 081001) showed lower significant detections in gamma-rays.

The large field of view of the GRID allows to simultaneously observe about one fifth of the Sky. Motivated by the fact that significant gamma-ray emission is observed from only a small fraction of GRBs, corresponding to few events per year taking into account the AGILE and Fermi/LAT detections, we estimated the upper limits on the flux of GRBs within the GRID field of view between July 2007 and October 2009, localised by SuperAGILE, Swift/BAT, INTEGRAL/IBIS, Fermi/GBM and IPN. Our sample is composed of 68 bursts, of which 40 have spectral information, publicly distributed through the GCN Circulars by Konus-Wind, Suzaku/WAM and Fermi/GBM. We estimated the upper limits using a Bayesian approach and following the method proposed by [16] and [17]. When available, the spectral model from the publicly available information is used to calculate the flux upper limit and to extrapolate the available flux to the energy band between 30 $\mathrm{MeV}$ and $3 \mathrm{GeV}$. In the other cases, we adopted for these calculations the average values measured 
by BATSE for the photon index of an exponential cutoff and for the high energy photon index of a Band function. We found that the calculated upper limits are constraining the extrapolation of the Band spectrum for $\sim 10 \%$ of the GRBs[18]. We are still investigating the consequences of such constraints on the emission models.

A remarkable case of this behaviour is the long GRB 090618, localised by Swift [19] and also by SuperAGILE (in a consistent position) and detected by the MCAL [20]. GRB 090618 is characterised by a remarkable fluence of $(3.2 \pm 0.6)^{-5} \mathrm{erg} \mathrm{cm}^{-2}$, measured by MCAL in the energy band between $350 \mathrm{keV}$ and $100 \mathrm{MeV}$. The GRB has a steep spectrum in the same energy band, with a photon index of -3.16 [20] by MCAL, and is not detected in gamma-rays by the GRID. The interested reader can find the details and the complete analysis of the upper limits of GRBs in the GRID in a forthcoming paper [18].

The ongoing observation of GRBs with AGILE and Fermi is showing that only a small subsample of events emits in gamma rays. In fact, the overall detection rate of both satellites is $\sim$ 10 bursts per year, consistent with current estimates [21]. From the data analysis it is emerging that GRBs emitting in the GeV band are also bright at lower energy. In fact, an analysis of eleven GRBs detected by Fermi/LAT until October 2009 shows that the fluence in Fermi/GBM ( $8 \mathrm{keV}$ $10 \mathrm{MeV}$ ) of these events belongs to the highest tail of the distribution [22]. From the AGILE and Fermi observations we can see that the GeV emission of GRBs is simultaneous to the prompt phase, often with a delayed onset and extended emission. Some events have the same spectral shape from $\mathrm{keV}$ up to $\mathrm{GeV}$ energies, modelled by a single Band function (for example GRB 080514B, [9]), while in other the gamma-ray emission is fitted by additional components, as for example in GRB 090510 [12], [13]) or GRB 090902B below $50 \mathrm{keV}$ and above $100 \mathrm{MeV}$ [23].

Up to now the afterglow of only two GRBs detected in gamma-rays, GRB 090401B [11] and GRB 090510 [14], has been observed by Swift with $\sim 120$ s delay after the prompt emission. In our analysis we investigated the GRID data of the sample of GRBs within our field of view and we did not find any significant gamma-ray afterglow emission until $3600 \mathrm{~s}$ after trigger [18.

\section{Observation of TGFs with AGILE}

Thanks to its flexible trigger logic on sub-millisecond time scales [7], MCAL proved to be a very efficient instrument for TGF detection. The average MCAL detection rate is $\sim 10$ TGFs/month, with the current severe selection criteria based on hardness ratio and fluence [24]. AGILE is one of the only 3 currently operative space instruments capable of detecting TGFs, together with RHESSI [25] and Fermi-GBM [26].

TGFs have been associated with strong thunderstorms mostly concentrated in the Earth's equatorial and tropical regions, at a typical altitude of $15-20 \mathrm{~km}$ [27]. The geographical and local-time distribution of the AGILE TGF sample detected between June, 2008, and January, 2010 shows evidence for events clustering on the African continental region and the south-east Asia. The geographical distribution, strongly peaked over continental areas, matches the distribution of lightnings over the equatorial region, confirming the association of the TGF phenomenon with thunderstorm activity. The local-time distribution, peaked over the mid-afternoon hours, reflect the thunderstorm activity over equatorial areas as well. The geographical and local-time distributions are in 
good agreement with those of the RHESSI sample, provided the same latitude interval is considered [24][28], strongly suggesting that the two instruments detect consistent populations.

The main AGILE discoveries in TGF science during two and a half years of observations are the following: the TGF spectrum extends at least up to $40 \mathrm{MeV}$ [24]; the high energy tail of the TGF spectrum is harder than expected and cannot be easily explained by previous theoretical models [29], based on bremsstrahlung emission in the atmospheric layers by a population of runaway electrons accelerated to relativistic energies by strong electric fields inside or above thunderclouds [30], [31], [32]; TGFs can be localized from space using the incoming direction of high-energy photons detected by the AGILE silicon tracker [33].

In the period between June 2008 and December 2009 the MCAL instrument triggered 119 bursts identified as TGFs according to the selection criteria discussed in [24]. For each of these bursts, the GRID dataset was searched for quasi-simultaneous gamma-ray events within a $200 \mathrm{~ms}$ time-window centered at the TGF start time T0, defined as the time of the first MCAL-photon associated with the TGF. A peak in the time-of-arrival distribution is evident for the $2 \mathrm{~ms}$ time bin immediately following $\mathrm{T} 0$. This peak includes 13 events, and the probability for it to be a statistical fluctuation (13 events or higher) is $6.5 \times 10^{-10}$ if we assume that GRID events are not correlated to TGFs and are distributed according to the Poisson law with the measured average rate of 5.1 counts/s. All these GRID events take place during the TGF emission time interval estimated from MCAL data only. Among these 13 GRID events, 9 of them have incoming direction compatible with the Earth, an average energy of $60 \mathrm{MeV}$, and can be directly associated to the TGF [33]. All TGFs in the selected sample have one associated GRID event except the remarkable case of TGF 12809-19, for which two closely spaced GRID events were detected. The incoming directions of the 9 selected events appear to be clustered close to the subsatellite point, with an average (maximum) $\phi$ angle (the angle between the photon direction and the satellite nadir) of $25.4^{\circ}\left(35.1^{\circ}\right)$ and distance to the subsatellite point of $260 \mathrm{~km}(390 \mathrm{~km})$. All 9 events are contained within a $1.14 \mathrm{sr}$ solid angle, a factor 3.4 smaller than the solid angle subtended by the Earth at the satellite altitude of $540 \mathrm{~km}$, which corresponds to a maximum visibility projected distance radius of $\sim 2600 \mathrm{~km}$ from the satellite footprint. If the GRID events originate directly from the TGF production site, these results are consistent with a distance to footprint less than $\sim 300 \mathrm{~km}$ estimated for RHESSI TGFs using sferics data [34][35],[36],[37].

\section{Conclusions}

Thanks to its capabilities, several important results on GRB and TGF science were obtained. AGILE detected few GRBs but representing all the main features present in GRB high energy domain, such us delayed onset, spectral extra-component and extended emission. The Upper limits that AGILE derived are useful to exclude the presence of an extra component at the same fluence level as the low energy emission in most of the GRB in its field of view. The detection of the high energy component of the TGF spectum and the first accurate localization of TGFs from space demonstrate the capability of the AGILE space mission to play a significant role also in TGF science. 


\section{References}

[1] M. Tavani et al., Nucl. Inst. and Meth. A, 588, 52 (2008)

[2] M. Tavani et al., $A \& A, \mathbf{5 0 2}, 995$ (2009)

[3] M. Feroci et al., Nucl. Inst. and Meth. A, 581, 728 (2007)

[4] M. Prest et al., Nucl. Instr. and Meth. A, 501, 280 (2003)

[5] A. Bulgarelli et al., Nucl. Instr. and Meth. A, 614, 213 (2010)

[6] C. Labanti, et al., Nucl. Instr. and Meth. A, 598, 470 (2009)

[7] F. Fuschino, et al., Nucl. Instr. and Meth. A, 588, 17 (2008)

[8] E. Del Monte et al., in Proceedings of SciNeGHE 2007, edited by A. Lionetto and A. Morselli, Frascati Physics Series, 45, 201, (2007)

[9] A. Giuliani et al., $A \& A$, 491, L25 (2008)

[10] A. Rossi et al., $A \& A$, 491, L29 (2008)

[11] F. Longo et al., in preparation, (2011)

[12] A. Giuliani et al., ApJL, 708, L84 (2010)

[13] M. Ackerman et al., ApJ, 716, 1178 (2010)

[14] M. De Pasquale et al., ApJL, 709, L146 (2010)

[15] E. Del Monte et al., in prep., (2011)

[16] O. Helene, Nucl. Instr. and Meth. A, 212, 319 (1983)

[17] O. Helene, Nucl. Instr. and Meth. A, 228, 120 (1984)

[18] F. Longo et al., $A \& A$ submitted, 2011

[19] P. Schady et al., GCN Circular, 9512 (2009)

[20] F. Longo et al., GCN Circular, 9524 (2009)

[21] D. Band et al., ApJ, 701, 1673 (2009)

[22] G. Ghisellini et al., MNRAS, 403, 926 (2010)

[23] A.A. Abdo et al., ApJL, 706, L138 (2009)

[24] M. Marisaldi et al., J. Geophys, Res., 115, A00E13 (2010)

[25] D.M. Smith et al., Science,307, 1085 (2005)

[26] M. Briggs et al., J. Geophys. Res., 115, A07323 (2010)

[27] J.R. Dwyer and D.M. Smith, Geophys. Res. Lett., 32 L22804 (2005)

[28] B.W. Grefenstette et al., J. Geophys. Res., 114, A02314 (2009)

[29] M. Tavani, et al., Phys. Rev. Lett., 106, 018501 (2011)

[30] A.V. Gurevich, G.M. Milich and R. Roussel-Dupre, Physics Letters A, 165, 463, (1992)

[31] J.R. Dwyer, Phys. Plasmas, 14, 042901 (2007)

[32] J.R. Dwyer, J. Geophys. Res., 113, D10103 (2008) 
[33] M. Marisaldi et al., Phys. Rev. Lett., 105, 128501 (2010)

[34] S.A. Cummer et al., Geophys. Res. Lett., 32, L08811 (2005)

[35] B.J Hazelton et al., Geophys. Res. Lett., 36, L01108 (2009)

[36] M.B. Cohen, et al., Geophys. Res. Lett., 37, L02801 (2010)

[37] V. Connaughton et al., J. Geophys, Res., in press (2011) 